\title{
Estimating annual prevalence of depression and anxiety disorder in multiple sclerosis using administrative data
}

\author{
Ruth Ann Marrie ${ }^{1,2,10^{*}} \mathbb{0}$, Randy Walld ${ }^{3}$, James M. Bolton ${ }^{4}$, Jitender Sareen ${ }^{4}$, John R. Walker ${ }^{5}$, Scott B. Patten ${ }^{6}$, \\ Alexander Singer ${ }^{7}$, Lisa M. Lix², Carol A. Hitchon'1, Renée El-Gabalawy ${ }^{5,8}$, Alan Katz ${ }^{2,3,7}$, John D. Fisk9 \\ Charles N. Bernstein ${ }^{1}$ and For the CIHR Team in Defining the Burden and Managing the Effects of Psychiatric \\ Comorbidity in Chronic Immunoinflammatory Disease
}

\begin{abstract}
Objective: Researchers have developed case definitions to estimate incidence and lifetime prevalence of depression and anxiety disorders in multiple sclerosis (MS) using administrative data. For policymakers however, the prevalence of a disease requiring ongoing treatment during a given period such as annual period prevalence may be more relevant for decision-making. We tested a case definition for annual period prevalence of depression and anxiety disorders in MS using administrative data.

Results: Using population-based administrative (health claims) data from Manitoba, Canada we identified 1922 persons with incident MS from 1989 to 2012, and 11,392 age, sex and geographically-matched controls from the general population. As compared to controls, MS patients had an elevated annual prevalence ratio of depression (1.77; 95\% confidence interval $[\mathrm{Cl}] 1.64,1.91)$, and anxiety disorders $(1.46 ; 95 \% \mathrm{Cl} 1.35,1.58)$. The annual prevalence of depression in our matched cohort was similar to that observed in the 2012 Canadian Community Health Survey, although the annual prevalence of anxiety was slightly higher. Administrative data can be used to estimate the annual period prevalence of psychiatric disorders in MS.
\end{abstract}

Keywords: Multiple sclerosis, Depression, Anxiety, Epidemiology

\section{Introduction}

The burden of psychiatric illness is high in multiple sclerosis (MS) due to its high prevalence [1], and adverse effects on outcomes such as hospitalizations and mortality $[2,3]$. In a systematic review, the prevalence of depression was $23.9 \%(95 \%$ CI $17.4,30.0 \%)$ and of anxiety disorders was $21.9 \%$ (95\% CI 8.76, 35.0) among those with MS [1]. Previously, we developed case definitions to identify psychiatric comorbidity in the MS population using administrative (health claims) data [4], and estimated the incidence and lifetime prevalence of psychiatric comorbidity. However, when planning health services,

\footnotetext{
*Correspondence: rmarrie@hsc.mb.ca

${ }^{10}$ Health Sciences Center, GF-543, 820 Sherbrook Street, Winnipeg, MB R3A 1R9, Canada

Full list of author information is available at the end of the article
}

the prevalence of a disease requiring ongoing treatment during a given period such as a year (i.e., annual period prevalence) is more useful than lifetime prevalence [5-7]. While health system changes may not reduce the lifetime prevalence of disease, annual prevalence could be reduced if effective treatments were provided and recurrences prevented $[8,9]$. Herein, we built on prior work [4] and tested an approach to measure annual period prevalence of depression and anxiety disorders in MS using administrative data.

\section{Main text \\ Setting}

This retrospective matched cohort study used administrative databases for Manitoba, Canada, held in the Manitoba Population Data Repository at the Manitoba Centre 
for Health Policy. Health care is publicly funded in Manitoba and, these databases prospectively capture health services for $>98 \%$ of the population. We used the Population Registry (which provided dates of birth, death and health care coverage, sex, and region of residence [postal code]); the Discharge Abstract Database (which provided hospitalizations, including admission and discharge dates, and up to 25 diagnoses recorded using International Classification of Disease [ICD]-9/10 codes); Medical Services (physician claims, which provided service date and single ICD-9-CM physician-coded diagnosis); and Drug Program Information Network (DPIN, which provided all community-dispensed prescriptions including drug name, dispensation date, and drug identification number [DIN]). DPIN became available in 1995, but all other databases were available from April 1, 1984-March 31,2012 . We linked these databases deterministically at the individual level using a de-identified unique personal health identification number.

\section{Study populations}

We applied a validated case definition to identify Manitoba residents with MS during the study period [10]. We classified individuals as having MS if they had $\geq 3$ hospital or physician or prescription claims for MS. Hospital or physician claims for MS were identified using ICD9/10 codes of 340/G35. Prescription claims for MS were identified using DINs for MS-specific disease-modifying therapies. We limited the analysis to incident cases of MS by removing cases with any demyelinating disease claims within 5 years before the index date. Given that the databases were available as of 1984, the earliest index date for an incident case was 1989. Next, a cohort matched 5:1 on sex, year of birth \pm 5 years, and forward sortation area (i.e. first 3 digits of postal code) was generated. As this was part of a larger study including other immune-mediated diseases [10], anyone with claims for demyelinating disease, inflammatory bowel disease, rheumatoid arthritis and related disorders were excluded. Each control was assigned the index date of its matched case.

\section{Psychiatric disorders}

We applied validated case definitions (Additional file 1: Table S1) to identify individuals who ever met definitions for depression or anxiety disorders (any anxiety disorder, not limited to generalized anxiety disorder) [4]. Since the case definitions for depression and anxiety disorder included prescription claims, we included a binary covariate in our analyses indicating whether the disorder occurred before or after prescription data became available in 1995 . We estimated lifetime prevalence by assuming that if an individual met the case definition for depression or an anxiety disorder, he or she remained affected thereafter, as long as he or she was still living in Manitoba. Our previous case definition validations used self-reported lifetime diagnoses of depression or anxiety disorders, and disorders ever recorded as present on medical records review as reference standards [4].

To estimate annual prevalence, once a person met the case definition, he or she was counted as an annual prevalent case if there were $\geq 2$ physician claims or one hospital claim for the psychiatric disorder in that year; for hospital claims the disorder had to be the most responsible diagnosis. Therefore an individual who stopped receiving care in a given year did not need to meet the full case definition to be counted as a case once care resumed. We did not consider prescription claims in the annual prevalence definition because of the frequent off-label use of antidepressants and anxiolytics in the MS population for symptom management [11]. To test these "annual" case definitions, we compared the annual prevalence estimates in the matched population to those reported for the general population in the Canadian Community Health Survey-Mental Health (CCHS$\mathrm{MH})$ [12]. In 2012, the CCHS-MH data $(\mathrm{N}=25,113)$ estimated the annual prevalence of depression and generalized anxiety disorders using the World Health Organization (WHO) Composite International Diagnostic Interview (CIDI), regardless of whether these conditions were diagnosed and treated.

\section{Covariates}

Covariates included sex (male as reference group), age (18-24 [reference group], 25-44, 45-64, $\geq 65$ ), socioeconomic status (SES) in quintiles (worst quintile as reference group), and region (urban or rural [reference group]). We linked participants' postal codes to dissemination-area level census data to determine SES as defined by the Socioeconomic Factor Index version 2 (SEFI-2). The SEFI-2 incorporates data regarding average household income, percent of single parent households, unemployment rate and high school education rate; scores $<0$ indicate better SES [13]. Winnipeg (population $>700,000$ ) and Brandon (population $>47,000$ ) were designated as urban regions. We included year in the regression models (below) to evaluate temporal trends.

\section{Analysis}

The prevalence estimates were age and sex-standardized to the 2010 Canadian population, and 95\% confidence intervals $(95 \% \mathrm{CI}$ ) were determined using a negative binomial distribution. We report age-specific prevalence estimates using age groups 18-24, 25-44, $45-64, \geq 65$ years, to be consistent with the CCHS-MH. We compared the annual prevalence estimates between the MS and matched cohorts using negative binomial 
regression models (to account for overdispersion) adjusting for covariates (above), for which we report prevalence ratios (PR) and 95\% CIs. We included the natural logarithm of person-years as the model offset to account for variable follow-up duration, and used generalized estimating equations with an exchangeable correlation structure to account for dependence of repeated prevalence estimates within individuals. In separate models, we tested if the average annual rate of change differed between the two cohorts by including an interaction term (cohort"year).

We performed statistical analyses using SAS V9.4 (SAS Institute Inc., Cary, NC).

\section{Results}

We identified 3514 incident cases of MS and 17,526 matched individuals without MS. The two cohorts were well-matched at the index date (Table 1).

In 2011, the crude lifetime prevalence of depression was $44.8 \%(95 \%$ CI $42.9,46.6 \%)$ in the MS cohort and
27.1\% (95\% CI 26.3, 27.8\%) in the matched cohort. The crude annual prevalence of depression in the MS cohort was $12.6 \%(95 \%$ CI $11.5,13.9 \%)$ and in the matched cohort was $7.7 \%$ (95\% CI 7.2, 8.1\%). The age and sexstandardized annual prevalence of depression was $10.7 \%$ (95\% CI 9.02, 12.6\%) in the MS cohort, 64\% higher (PR $1.64 ; 95 \%$ CI $1.35,1.98)$ than in the matched cohort $(6.5 \%$; 95\% CI 5.9, 7.2\%).

The annual prevalence of depression was lower than the lifetime prevalence of depression at all ages (Table 2). After accounting for differences in the sex distribution of our matched population and the Canadian general population, the annual prevalence of depression in the Canadian general population, as drawn from the CCHS-MH, was similar to that observed in our matched cohort in all groups except those aged $\geq 65$ years (Table 2 ).

After adjustment for age, sex, SES, region of residence and year, the annual prevalence of depression (IRR 1.76; $95 \%$ CI 1.62, 1.90) was higher in the MS cohort than the matched cohort over the entire study period (Additional

Table 1 Characteristics of incident multiple sclerosis (MS) and matched cohorts at the index date

\begin{tabular}{|c|c|c|}
\hline Characteristic & MS matches $(n=17,526)$ & MS $(n=3514)$ \\
\hline Female, n (\%) & $12,697(72.4)$ & $2544(72.4)$ \\
\hline Age at diagnosis, mean (SD) & $40.8(12.5)$ & $40.8(12.5)$ \\
\hline Duration of follow-up from the index date (years), median (IQR) & $10.5(5.0,16.3)$ & $10.3(4.90,16.1)$ \\
\hline \multicolumn{3}{|l|}{ Region of residence, $\mathrm{n}(\%)$} \\
\hline Urban & $11,685(66.7)$ & $2344(66.7)$ \\
\hline Rural & $5841(33.3)$ & $1170(33.3)$ \\
\hline Socioeconomic Factor Index Score ${ }^{a}$ & $-0.18(0.87)$ & $-0.24(0.89)$ \\
\hline
\end{tabular}

a Values less than zero indicate higher socioeconomic status

Table 2 Age-specific prevalence (95\% confidence interval) of depression and anxiety disorders per 100 population in 2011

\begin{tabular}{|c|c|c|c|c|c|c|c|c|}
\hline \multicolumn{5}{|l|}{ Annual } & \multirow{2}{*}{$\begin{array}{l}\text { Prevalence } \\
\text { ratio MS: } \\
\text { matches }\end{array}$} & \multicolumn{2}{|l|}{ Lifetime } & \multirow{2}{*}{$\begin{array}{l}\text { Prevalence ratio } \\
\text { MS: matches }\end{array}$} \\
\hline Age group & MS & Matches & CCHS-MH ${ }^{a}$ & $\begin{array}{l}\text { CCHS-MH } \\
\text { reweighted }^{b}\end{array}$ & & MS & Matches & \\
\hline \multicolumn{9}{|l|}{ Depression } \\
\hline $18-24$ & $12.1(7.3,16.8)$ & $8.3(6.5,10.1)$ & $7.1^{c}(5.9,8.3)$ & $8.0^{c}(5.9,10.1)$ & $1.45^{\mathrm{d}}(0.93,2.27)$ & $37.2(28.9,45.5)$ & $23.6(20.6,26.6)$ & $1.58^{f}(1.22,2.04)$ \\
\hline $25-44$ & $13.0(11.3,14.8)$ & $8.1(7.5,8.7)$ & $5.4(4.6,6.3)$ & $6.1(4.3,7.9)$ & $1.61^{\mathrm{e}}(1.38,1.87)$ & $45.7(42.5,48.9)$ & $27.9(26.8,29.0)$ & $1.64^{\mathrm{e}}(1.51,1.77)$ \\
\hline $45-64$ & $11.7(9.4,14.0)$ & $6.7(5.9,7.4)$ & $4.5(3.9,5.1)$ & $5.0(3.5,6.5)$ & $1.76^{\mathrm{e}}(1.40,2.22)$ & $45.4(40.8,50.0)$ & $26.5(24.9,28.0)$ & $1.72^{\mathrm{e}}(1.53,1.93)$ \\
\hline$\geq 65$ & s & $6.5(3.9,9.0)$ & $1.6(1.2,2.1)$ & $1.7(0.6,2.8)$ & $1.45^{d}(0.93,2.27)$ & $36.8(21.1,52.6)$ & $24.0(19.1,28.9)$ & $1.53^{9}(0.95,2.46)$ \\
\hline \multicolumn{9}{|c|}{ Anxiety disorder } \\
\hline $18-24$ & $5.8(2.5,9.1)$ & $7.6(5.9,9.3)$ & $2.4(1.7,3.1)$ & $3.1(1.4,4.8)$ & $0.76^{h}(0.41,1.40)$ & $43.0(34.1,51.9)$ & $33.6(30.0,37.2)$ & $1.28^{\prime}(1.01,1.62)$ \\
\hline $25-44$ & $6.3(5.1,7.5)$ & $5.4(4.9,5.9)$ & $2.9(2.4,3.4)$ & $3.1(1.7,4.5)$ & $1.18^{i}(0.96,1.46)$ & $50.0(46.6,53.4)$ & $37.9(36.6,39.2)$ & $1.32^{\mathrm{e}}(1.22,1.42)$ \\
\hline $45-64$ & $6.1(4.4,7.8)$ & $4.7(4.0,5.3)$ & $3.0(2.5,3.5)$ & $3.4(2.0,4.8)$ & $1.31^{\mathrm{j}}(0.96,1.79)$ & $48.4(43.6,53.1)$ & $39.0(37.2,40.9)$ & $1.24^{\mathrm{e}}(1.11,1.38)$ \\
\hline$\geq 65$ & $\mathrm{~s}$ & $3.1(1.3,4.9)$ & $1.2(0.8,1.6)$ & $1.2(0.1,2.3)$ & $0.57^{k}(0.07,4.35)$ & $47.4(29.5,65.2)$ & $38.5(32.3,44.7)$ & $1.23^{\mathrm{m}}(0.82,1.85)$ \\
\hline
\end{tabular}

${ }^{a}$ Canadian Community Health Survey-Mental Health (CCHS-MH) estimates; ${ }^{b}$ CCHS-MH estimates re-weighted to match male-female proportion in the matched study population; ${ }^{\mathrm{C} C C H S}-\mathrm{MH}$ age group is $15-24$ years; $s$ suppressed due to privacy and confidentiality considerations; ${ }^{\mathrm{d}} \mathrm{p}=0.10 ;{ }^{\mathrm{e}} \mathrm{p}<0.0001 ;{ }^{\mathrm{f}} \mathrm{p}=0.0005$; ${ }^{g} p=0.077 ;{ }^{h} p=0.38 ;{ }^{i} p=0.12 ; p=0.088 ;{ }^{k} p=0.58 ;{ }^{i} p=0.038 ;{ }^{m} p=0.32$ 
file 1: Table S1). Female sex, older age (versus persons aged 18-24 years), urban versus rural residence, and lower SES were associated with an increased prevalence of depression. The lifetime and annual prevalence increased slightly over time, but less in the MS than the matched cohort (both $\mathrm{p}<0.0001$, Fig. 1).

In 2011, the crude lifetime prevalence of anxiety was 48.9\% (95\% CI 47.1, 50.8\%) in the MS cohort and 37.9\% (95\% CI 37.1, 38.7\%) in the matched cohort. The crude annual prevalence of anxiety disorders in the MS cohort was $6.2 \%$ (95\% CI 5.3, 7.1\%) and in the matched cohort was 5.3\% (95\% CI 5.7, 6.0\%). The age- and sex-standardized annual prevalence of anxiety disorders in the MS cohort was $5.6 \%$ (95\% CI 4.6, 6.9\%), 13\% higher (1.13; 95\% CI 0.90, 1.42) than in the matched cohort (5.0\%; 95\% CI 4.4, 5.6\%).

Annual prevalence was lower than lifetime prevalence for anxiety disorders in all age groups (Table 2). After accounting for differences in the sex distribution of our matched population and the Canadian general population, the annual prevalence of anxiety in the Canadian general population, as drawn from the CCHS-MH, was slightly lower than that observed in our matched cohort in all groups except those aged $\geq 65$ years (Table 2 ).

After adjustment for age, sex, SES, region of residence and year, the annual prevalence of anxiety disorder (PR 1.46 ; $95 \%$ CI $1.35,1.58$ ) was higher in the MS cohort than the matched cohort over the entire study period (Additional file 1: Table S1). Female sex, older age (as compared to persons aged 18-24 years), urban versus rural residence, and lower SES were associated with an increased prevalence of anxiety. The lifetime and annual prevalence increased slightly over time, but less in the MS than the matched cohort (both $\mathrm{p}<0.0001$ ).

\section{Discussion}

In this population-based study the lifetime and annual prevalence of depression and anxiety disorders were higher in the MS population than in a matched general

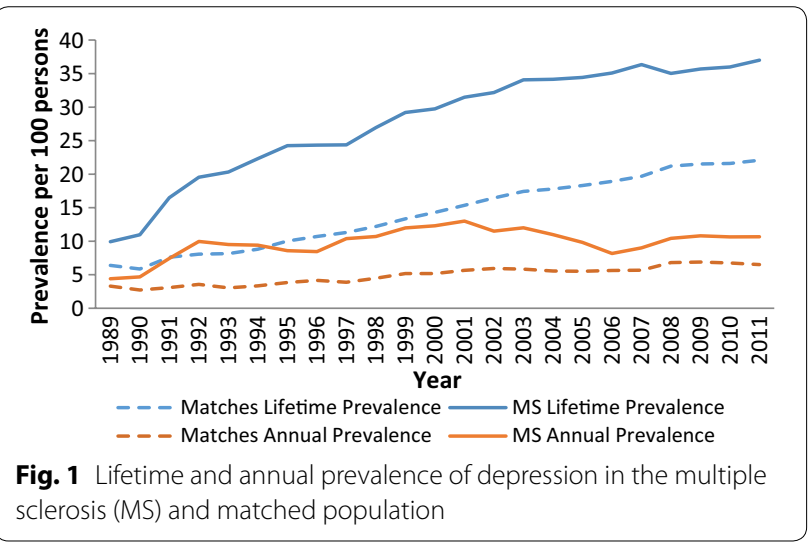

population cohort without MS. In the MS population, the annual period prevalence of depression and anxiety disorders changed minimally over time, suggesting consistent patterns of health care utilization for those conditions over time. Accounting for the higher proportion of women in our sample than in the CCHS-MH sample, age-specific annual prevalence estimates of depression in our matched cohort fell within the bounds of the CCHS-MH estimates for all age groups except age $\geq 65$ years. Our age-specific anxiety disorder estimates were slightly higher than those reported in the CCHS-MH, which may reflect the fact that the CCHS-MH identified only generalized anxiety disorder, while our case definition captured anxiety disorders more generally. Overall, the findings suggest that our proposed approach to identifying annual period prevalence is appropriate and could be applied to other conditions. Comparable work is limited. The Canadian Chronic Disease Surveillance System identifies annual prevalence of mental illness on the basis of any hospitalization or physician visit for mental illness but this case definition includes dementia and developmental disorders, and does not attempt to distinguish diagnoses due to feasibility concerns $[1,2]$. Other investigators have developed administrative case definitions for depression or anxiety, but have not explicitly distinguished annual and lifetime prevalence [3, 4].

Several demographic factors were associated with the prevalence of depression and anxiety. Women had an increased prevalence of both disorders, consistent with prior studies in MS [14]. As reported in the general population, urban residence was associated with an increased prevalence of depression [15]. Lower SES was associated with an increased prevalence of depression and anxiety, consistent with prior studies showing that lower annual household income is associated with an increased lifetime prevalence of depression in MS [16], and that persons with MS with lower levels of education had greater depressive symptoms [17].

\section{Limitations}

Administrative data may be subject to diagnostic misclassification bias. However, we employed a validated case definition for MS, and the case definitions used to identify lifetime depression and anxiety disorder were developed and validated in two MS populations $[4,18]$. We did not validate our case definitions for annual prevalence of depression or anxiety disorders at the individual level, but rather used population-level comparisons. Nonetheless, we identified the expected demographic relationships with the psychiatric disorders studied. Study strengths include the large study population, and use of population-based data sources and concurrent controls.

Administrative data can be used to estimate annual period prevalence of psychiatric disorders. The 
prevalence of depression and anxiety disorders is higher in the MS cohort than in an age-, sex- and geographically-matched cohort without MS.

\section{Additional file}

Additional file 1: Table S1. Adjusted rate ratios and $95 \%$ confidence intervals for the association between multiple sclerosis (MS) and prevalence of depression and anxiety disorder. Figure S1. Lifetime and annual prevalence of anxiety in the multiple sclerosis (MS) and matched populations.

\section{Authors' contributions}

RAM and CB conceived of the initial study design. RAM, JMB, JS, JRW, SBP, AS, $L M L, C A H, R E G, A K, J D F, C N B$ authors contributed to the design of the study protocol. RAM, CB, JMB, JS, JRW, SBP, LML, CAH, REG, AK, JDF obtained study funding. RAM, CB contributed to data acquisition. RW analyzed the data. RAM drafted the manuscript. RAM, RW, JMB, JS, JRW, SBP, AS, LML, CAH, REG, $A K, J D F, C N B$ revised the manuscript. All authors read and approved the final manuscript.

\section{Author details}

${ }^{1}$ Department of Internal Medicine, Max Rady College of Medicine, Rady Faculty of Health Sciences, University of Manitoba, Winnipeg, Canada. ${ }^{2}$ Department of Community Health Sciences, Max Rady College of Medicine, Rady Faculty of Health Sciences, University of Manitoba, Winnipeg, Canada. ${ }^{3}$ Manitoba Centre for Health Policy, Max Rady College of Medicine, Rady Faculty of Health Sciences, University of Manitoba, Winnipeg, Canada. ${ }^{4}$ Department of Psychiatry, Max Rady College of Medicine, Rady Faculty of Health Sciences, University of Manitoba, Winnipeg, Canada. ${ }^{5}$ Department of Clinical Health Psychology, Max Rady College of Medicine, Rady Faculty of Health Sciences, University of Manitoba, Winnipeg, Canada. ${ }^{6}$ Department of Community Health Sciences, Cumming School of Medicine, University of Calgary, Calgary, Canada. ${ }^{7}$ Department of Family Medicine, Max Rady College of Medicine, Rady Faculty of Health Sciences, University of Manitoba, Winnipeg, Canada. ${ }^{8}$ Department of Anesthesia and Perioperative Medicine, Max Rady College of Medicine, Rady Faculty of Health Sciences, University of Manitoba, Winnipeg, Canada. ${ }^{9}$ Departments of Psychiatry, Psychology \& Neuroscience, and Medicine, Dalhousie University, Halifax, Canada. ${ }^{10}$ Health Sciences Center, GF-543, 820 Sherbrook Street, Winnipeg, MB R3A 1R9, Canada.

\section{Acknowledgements}

Members of the ClHR Team in Defining the Burden and Managing the Effects of Psychiatric Comorbidity in Chronic Immunoinflammatory Disease are: Ruth Ann Marrie, James M Bolton, Jitender Sareen, John R Walker, Scott B Patten, Alexander Singer, Lisa M. Lix, Carol A Hitchon, Renée El-Gabalawy, Alan Katz, John D Fisk, Charles N Bernstein, Lesley Graff, Lindsay Berrigan, Ryan Zarychanski, Christine Peschken, James Marriott.

\section{Competing interests}

Ruth Ann Marrie has conducted clinical trials for Sanofi Aventis. Carol Hitchon has research funds for unrelated studies from UCB Canada. Jitender Sareen holds stocks in Johnson and Johnson. Charles Bernstein has consulted to Abbvie Canada, Ferring Canada, Janssen Canada, Pfizer Canada, Shire Canada, Takeda Canada, and Napo Pharmaceuticals and has consulted to Mylan Pharmaceuticals. He has received unrestricted educational grants from Abbvie Canada, Janssen Canada, Shire Canada, and Takeda Canada. He has been on speaker's bureau of Abbvie Canada, Ferring Canada and Shire Canada. All other authors declare that they have no competing interests.

\section{Availability of data and materials}

We are unable to make the dataset used in this study publicly available as we are not the custodians of the data, but rather obtained permission to access the data for this project.

\section{Consent for publication}

Not applicable.

\section{Ethics approval and consent to participate}

We obtained ethics approvals from the University of Manitoba Health Research Ethics Board, and approval to access administrative data from the Health Information Privacy Committee (Province of Manitoba). The authors acknowledge the Manitoba Centre for Health Policy for use of the Manitoba Population Research Data Repository under project \#2014-030 (HIPC \#2015/2015-19A). The results and conclusions presented are those of the authors and no official endorsement by the Manitoba Centre for Health Policy, Manitoba Health Seniors and Active Living, or other data providers is intended or should be inferred.

\section{Funding}

This study was funded by the Canadian Institutes of Health Research (THC135234), Crohn's and Colitis Canada, and the Waugh Family Chair in Multiple Sclerosis (to RAM). Dr. Bernstein is supported in part by the Bingham Chair in Gastroenterology. Dr. Sareen is supported by CIHR \#333252. Dr. Katz is supported by Research Manitoba and the Health and Stroke Foundation through the Manitoba Chair in Primary Prevention Research. Dr. Lix is supported by a Research Manitoba Chair. Dr. Zarychanski is supported by a CIHR New Investigator Salary Award. The sponsors had no role in the design and conduct of the study; collection, management, analysis, and interpretation of the data; and preparation, review, or approval of the manuscript.

\section{Publisher's Note}

Springer Nature remains neutral with regard to jurisdictional claims in published maps and institutional affiliations.

Received: 19 September 2017 Accepted: 21 November 2017

Published online: 25 November 2017

\section{References}

1. Marrie R, Reider N, Cohen J, Stuve O, Trojano M, Sorensen PS, Cutter G. The incidence and prevalence of psychiatric disorders in multiple sclerosis: a systematic review. Mult Scler J. 2015;21(3):305-17.

2. Marrie RA, Elliott L, Marriott J, Cossoy M, Blanchard J, Leung S, Yu N. Effect of comorbidity on mortality in multiple sclerosis. Neurology. 2015:85(3):240-7.

3. Marrie RA, Elliott L, Marriott J, Cossoy M, Tennakoon A, Yu N. Comorbidity increases the risk of hospitalizations in multiple sclerosis. Neurology. 2015:84(4):350-8.

4. Marrie RA, Fisk JD, Yu BN, Leung S, Elliott L, Caetano P, Warren S, Evans C, Wolfson C, Svenson LW, et al. Mental comorbidity and multiple sclerosis: validating administrative data to support population-based surveillance. BMC Neurol. 2013;13(1):16.

5. Baxter AJ, Vos T, Scott KM, Norman RE, Flaxman AD, Blore J, Whiteford HA. The regional distribution of anxiety disorders: implications for the Global Burden of Disease Study, 2010. Int J Methods Psychiatr Res. 2014:23(4):422-38.

6. Ferrari AJ, Charlson FJ, Norman RE, Patten SB, Freedman G, Murray CJL, Vos T, Whiteford HA. Burden of depressive disorders by country, sex, age, and year: findings from the global burden of disease study 2010. PLoS Med. 2013;10(11):e1001547.

7. World Health Organization. Mental Health Action Plan 2013-2020. Geneva: World Health Organization; 2013.

8. Keller MB, Boland RJ. Implications of failing to achieve successful longterm maintenance treatment of recurrent unipolar major depression. Biol Psychiatr. 1998:44(5):348-60.

9. Bockting CL, Hollon SD, Jarrett RB, Kuyken W, Dobson K. A lifetime approach to major depressive disorder: the contributions of psychological interventions in preventing relapse and recurrence. Clin Psychol Rev. 2015:41:16-26.

10. Marrie RA, Walld R, Bolton JM, Sareen J, Walker JR, Patten SB, Singer A, Lix $L M$, Hitchon CA, El-Gabalawy H, et al. Increased incidence of psychiatric disorders in immune-mediated inflammatory disease. J Psychosom Res. 2017;101:17-23. 
11. Goldman MD, Cohen JA, Fox RJ, Bethoux FA. Multiple sclerosis: treat ing symptoms, and other general medical issues. Cleve Clin J Med. 2006;73:177-86

12. Canadian Community Health Survey-Mental Health (CCHS). http:// www23.statcan.gc.ca/imdb/p2SV.pl?Function=getSurvey\&ld $=119789$. Accessed 11 Sep 2017.

13. Chateau D, Metge C, Prior $H$, Soodeen RA. Learning from the census: the Socio-economic Factor Index (SEFI) and health outcomes in Manitoba. Can J Public Health. 2012;103(8 Suppl 2):S23-7.

14. Patten SB, Svenson LW, Metz LM. Descriptive epidemiology of affective disorders in multiple sclerosis. CNS Spectr. 2005;10(5):365-71.

15. Breslow RE, Klinger BI, Erickson BJ. County drift: a type of geographic mobility of chronic psychiatric patients. Gen Hosp Psychiatry. 1998;20(1):44-7.
16. Marrie RA, Horwitz RI, Cutter G, Tyry T, Campagnolo D, Vollmer T. The burden of mental comorbidity in multiple sclerosis: frequent, underdiagnosed, and under-treated. Neurology. 2008;70(Suppl 1):A208.

17. Chwastiak L, Ehde DM, Gibbons LE, Sullivan M, Bowen JD, Kraft GH. Depressive symptoms and severity of illness in multiple sclerosis: epidemiologic study of a large community sample. Am J Psychiatry. 2002;159(11):1862-8

18. Marrie RA, Walker JR, Graff LA, Lix LM, Bolton JM, Nugent Z, Targownik $L E$, Bernstein CN. Performance of administrative case definitions for depression and anxiety in inflammatory bowel disease. J Psychosom Res. 2016;89:107-13.

\section{Submit your next manuscript to BioMed Central and we will help you at every step:}

- We accept pre-submission inquiries

- Our selector tool helps you to find the most relevant journal

- We provide round the clock customer support

- Convenient online submission

- Thorough peer review

- Inclusion in PubMed and all major indexing services

- Maximum visibility for your research

Submit your manuscript at www.biomedcentral com/submit 